\title{
Successive metamagnetic transitions and magnetoresistance in the low-carrier-density strongly correlated electron system CeP
}

\author{
T. Terashima, ${ }^{*}$ S. Uji, and H. Aoki \\ Tsukuba Magnet Laboratory, National Research Institute for Metals, Tsukuba, Ibaraki 305, Japan \\ J. A. A. J. Perenboom \\ Research Institute for Materials and High Field Magnet Laboratory, University of Nijmegen, NL-6525 ED, Nijmegen, The Netherlands
}

Y. Haga

Advanced Science Research Center, Japan Atomic Energy Research Institute, Tokai, Ibaraki 319-11, Japan

A. Uesawa and T. Suzuki

Department of Physics, Tohoku University, Sendai 980-77, Japan

S. Hill and J. S. Brooks

National High Magnetic Field Laboratory, Tallahassee, Florida 32310

(Received 12 December 1997)

\begin{abstract}
Successive metamagnetic transitions in $\mathrm{CeP}$ are studied through magnetoresistance measurements up to 31.5 $\mathrm{T}$ over a wide temperature range between 0.7 and $44.1 \mathrm{~K}$. As in previous papers, we find a series of transitions in field and temperature that constitute a phase diagram. However, we find the observed transitions not to be periodic in inverse field, and hence our results do not support previous models where the transitions are triggered by crossings of the up- and down-spin Landau levels in a particular electronic energy band. The present phase diagram reveals a close similarity to that of $\mathrm{CeSb}$, and its general features are satisfactorily explained with a simple thermodynamic model. [S0163-1829(98)01425-8]
\end{abstract}

\section{INTRODUCTION}

The semimetallic cerium monopnictides $\mathrm{Ce} X(X=\mathrm{P}$, As, $\mathrm{Bi}$, and $\mathrm{Sb}$ ) with the $\mathrm{NaCl}$ structure have been an object of extensive studies for many years. ${ }^{1,2}$ The carrier concentration in these compounds is very low, a few \%/Ce or less, and the strong interplay between the carriers and the Ce $4 f$ spin system leads to highly unusual magnetic and transport properties such as the complex magnetic phase diagram of $\mathrm{CeSb}^{2}$ Compared with $\mathrm{CeSb}$, the title compound $\mathrm{CeP}$ was thought to be rather conventional. However, recent detailed investigations of $\mathrm{CeP}$, made possible by successful growth of highquality single crystals, have revealed that $\mathrm{CeP}$ is as rich as $\mathrm{CeSb}$ in the anomalous physical properties. ${ }^{3,4}$

Figure 1 shows a schematic magnetic field versus temperature $(H-T)$ phase diagram of $\mathrm{CeP}$ at low temperatures and low magnetic fields applied parallel to $[001] .^{4}$ In the paramagnetic state, the crystal-field ground state of the $\mathrm{Ce}^{3+}$ ion is $\Gamma_{7}$ with the excited $\Gamma_{8}$ state about $150 \mathrm{~K}$ above. ${ }^{5}$ At zero magnetic field $\mathrm{CeP}$ orders antiferromagnetically below $T_{N}=10.5 \mathrm{~K}$ with a simple type-I structure. The ordered moment, which is parallel to the $\langle 001\rangle$ axis, is $0.8 \mu_{B}$, close to the value expected for the $\Gamma_{7}$ ground state. ${ }^{6}$ With the magnetic field applied, three distinct regions called phases I, II, and III appear in the phase diagram. Neutron-scattering experiments have shown that two kinds of the Ce spins coexist in these regions (Fig. 1): one is the $\Gamma_{7}$ spin with a magnetic moment of $0.7 \mu_{B}$, while the other is the $\Gamma_{8}$ spin with $2 \mu_{B} \cdot{ }^{6}$ The spin structure in phase $\mathrm{I}$ is an eleven layer one consisting of a ferromagnetically coupled double layer of the
$\Gamma_{8}$ spins and antiferromagnetically coupled nine layers of the $\Gamma_{7}$ spins. The spins are ferromagnetically coupled within each layer. On entering phase II with increasing field, the $\Gamma_{7}$ spins are canted. In phase III, the $\Gamma_{7}$ spins are paramagnetic and the period of the spin structure observed at $5.3 \mathrm{~T}$ is ten layers. ${ }^{6}$ It is an intriguing problem why the $\Gamma_{8}$ state is stabilized in these phases despite the large crystal-field separation. To answer this, Kasuya and co-workers argue formation of magnetic polarons based on the $p-f$ mixing model. ${ }^{7}$

The most mysterious phenomenon found in CeP is successive metamagnetic transitions occurring at high magnetic fields above $20 \mathrm{~T}^{8}{ }^{8}$ They were observed as steps in magnetization curves recorded with pulsed magnetic fields. The transition fields were reported to be equally spaced on the scale of the inverse field $(1 / H)$. By analogy with the ordinary de Haas-van Alphen (dHvA) effect, the transition frequency $F_{\text {meta }}$ may be defined from the spacing of the transitions as $F_{\text {meta }}=[\Delta(1 / H)]^{-1}$. For the field direction [001], $F_{\text {meta }}$ agreed well with a frequency observed in early Shubnikov-de Haas ( $\mathrm{SdH})$ effect measurements. To explain the phenomenon, Kuroda et al. proposed the Stoner-Landau model, in which the metamagnetic transitions are triggered by crossings of up- and down-spin Landau levels in the particular electronic energy band whose Fermi surface was seen via the $\mathrm{SdH}$ oscillation. ${ }^{8}$ In previous $\mathrm{dHvA}$ effect measurements below $14 \mathrm{~T}$, we also confirmed that $F_{\text {meta }}$ agrees well with one of the dHvA frequencies $\alpha_{1}$, observed in phase II for all directions of the magnetic field. ${ }^{9}$ However, our recent dHvA effect measurements up to $20 \mathrm{~T}$ cast some doubt about such correlation between the metamagnetic transitions and 


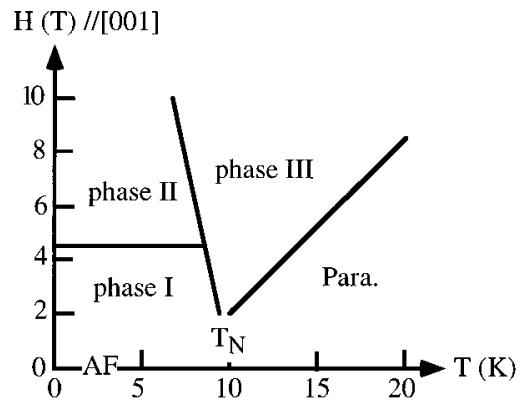

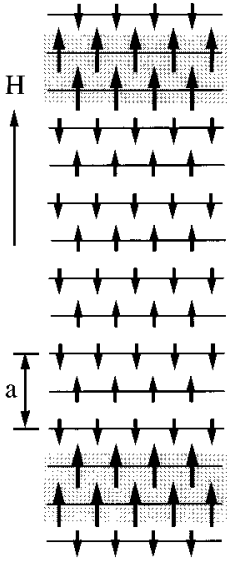

phase I

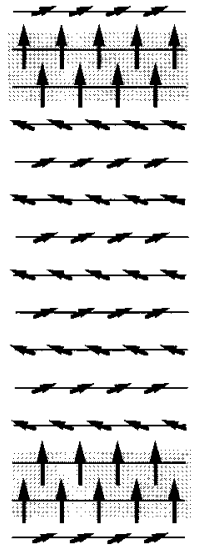

phase II

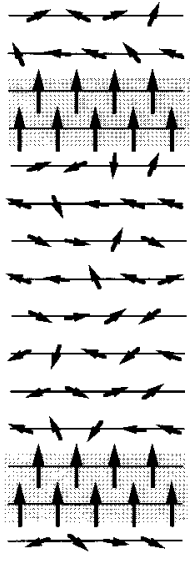

phase III
FIG. 1. Simplified magnetic phase diagram for magnetic fields below $10 \mathrm{~T}$ applied parallel to the [001] axis of CeP (based on Ref. 4) and spin structures determined by neutron-scattering experiments (Ref. 6). The long and short arrows indicate the $\Gamma_{8}$ and $\Gamma_{7}$ spins, respectively.

the Fermi surface. In a higher-field phase than phase II, the $\alpha_{1}$ frequency corresponding to $F_{\text {meta }}$ can be detected only for limited field directions around [001]. ${ }^{10}$ If the $\alpha_{1}$ frequency were directly connected with the successive metamagnetic transitions, it should be observed for all field directions.

In this work, we have studied the successive metamagnetic transitions through magnetoresistance measurements up to $31.5 \mathrm{~T}$ over a wide temperature range. The phase diagram thus obtained shows appreciable disagreement at low temperatures with that of a previous work based on pulsed-field magnetization measurements. Our data do not favor a correlation between the successive metamagnetic transitions and the Landau quantization of the conduction-electron energy: the positions of the transition fields are not equally spaced on the $1 / H$ scale. Rather, the data clearly indicate a close relation between the successive metamagnetic transitions in CeP and the ferroparamagnetic (FP) phase of CeSb (Ref. 2).

\section{EXPERIMENTAL PROCEDURES AND RESULTS}

The single crystal of CeP used in this study was grown by a recrystallization method as described in Ref. 3 . The magnetoresistance in magnetic fields up to $31.5 \mathrm{~T}$ was measured between 0.7 and $44.1 \mathrm{~K}$ with the standard four contact method using an ac current $(f=100 \mathrm{~Hz})$. The magnetic field was applied in the [001] direction and was parallel to the current (longitudinal configuration). For magnetic field ramping, a slow sweep rate of about $0.04 \mathrm{~T} / \mathrm{s}$ was normally used to minimize the eddy current heating. The sample and a Cernox resistance thermometer were mounted as closely as possible on the same copper plate to eliminate temperature differences between them. Temperature control below $4 \mathrm{~K}$ was done by stabilizing ${ }^{4} \mathrm{He}$ or ${ }^{3} \mathrm{He}$ pressure, while above 4 $\mathrm{K}$ a heater was employed using a PID temperature controller with the Cernox thermometer. In the latter case, the temperature error due to the magnetoresistance of the sensor is estimated to be a few $\%$ between 4 and $15 \mathrm{~K}$, and less than $1 \%$ above $15 \mathrm{~K} .{ }^{11}$ To avoid complications due to magnetic hysteresis, the magnetoresistance measurements below $T_{N}$ were always made after heating the sample above $T_{N}$ and cooling it in zero magnetic field.

We show the magnetoresistance at $0.7 \mathrm{~K}$ in Fig. 2(a) as a typical magnetoresistance trace observed below $T_{N}$. With increasing field, the resistivity starts to increase sharply at about $0.3 \mathrm{~T}$, reaches the maximum at $H_{p}=0.9 \mathrm{~T}$, and then decreases. Small features appear at 2.4 and 3.6 T. The resistivity drops further at $H_{f}=4.6 \mathrm{~T}$ and $H_{c}=14.5 \mathrm{~T}$. The oscillations in the resistivity between $H_{f}$ and $H_{c}$ are due to the $\mathrm{SdH}$ effect. No clear transition appears above $H_{c}$. However, apparent hysteresis is observed between the up- and downfield-sweep traces. The single $H_{f}$ transition in the up-sweep trace splits into two successive transitions, $H_{f}$ at $4.6 \mathrm{~T}$ and $H_{f^{\prime}}$ at $3.6 \mathrm{~T}$, in the down-sweep trace. The resistivity peak does not appear in the down-sweep trace.

Figure 2(b) shows the magnetoresistance at $T=23.3 \mathrm{~K}$ $\left(>T_{N}\right)$. On entering the phase III region at the magnetic field $H_{0}$, the resistivity decreases sharply. Above $H_{0}$, five distinct transitions from $H_{1}$ to $H_{5}$, all accompanied by a resistivity decrease, appear with a broad hysteresis. They correspond to the successive metamagnetic transitions observed in the magnetization. Figure 2(c) shows the magnetoresistance traces as a function of temperature above $T_{N}$. (Note that we used a higher sweep rate to record the $T=44.1 \mathrm{~K}$ trace. Therefore, the hysteresis in the $T=44.1 \mathrm{~K}$ trace, which appears to persist to very low fields, is most likely due to a time delay caused by the time constant of a lock-in amplifier.) With increasing temperature, the transition field $H_{0}$ increases rapidly and the accompanying resistivity decrease becomes less sharp. It seems that the $H_{0}$ transition changes from first order to second order at about $40 \mathrm{~K}$. At low temperatures, the hysteresis of the successive metamagnetic transitions $H_{1}-H_{5}$ are remarkably large, extending for more than $5 \mathrm{~T}$ for the $H_{5}$ transition at $11.0 \mathrm{~K}$. The hysteresis becomes smaller with increasing temperature, but is still prominent even at $T=39.5 \mathrm{~K}$. The transition fields show a clear but nonmonotonic temperature variation: they first shift to higher fields, reach a maxima at about $30 \mathrm{~K}$, then decrease.

Because of the temperature dependence of the successive metamagnetic transitions, the transitions are also visible in temperature variation of the resistivity at a constant field. In the $H=23 \mathrm{~T}$ resistivity trace shown in the inset of Fig. 2(a), the broad hysteresis extending between about 20 and $28 \mathrm{~K}$ is due to the $\mathrm{H}_{4}$ transition.

Figure 3 summarizes all the transitions and anomalies observed in the resistivity versus magnetic-field traces. Note that all the transitions $H_{0}-H_{5}$, except $H_{0}$ above about $40 \mathrm{~K}$, are of first order judging from their apparent hysteresis. The 

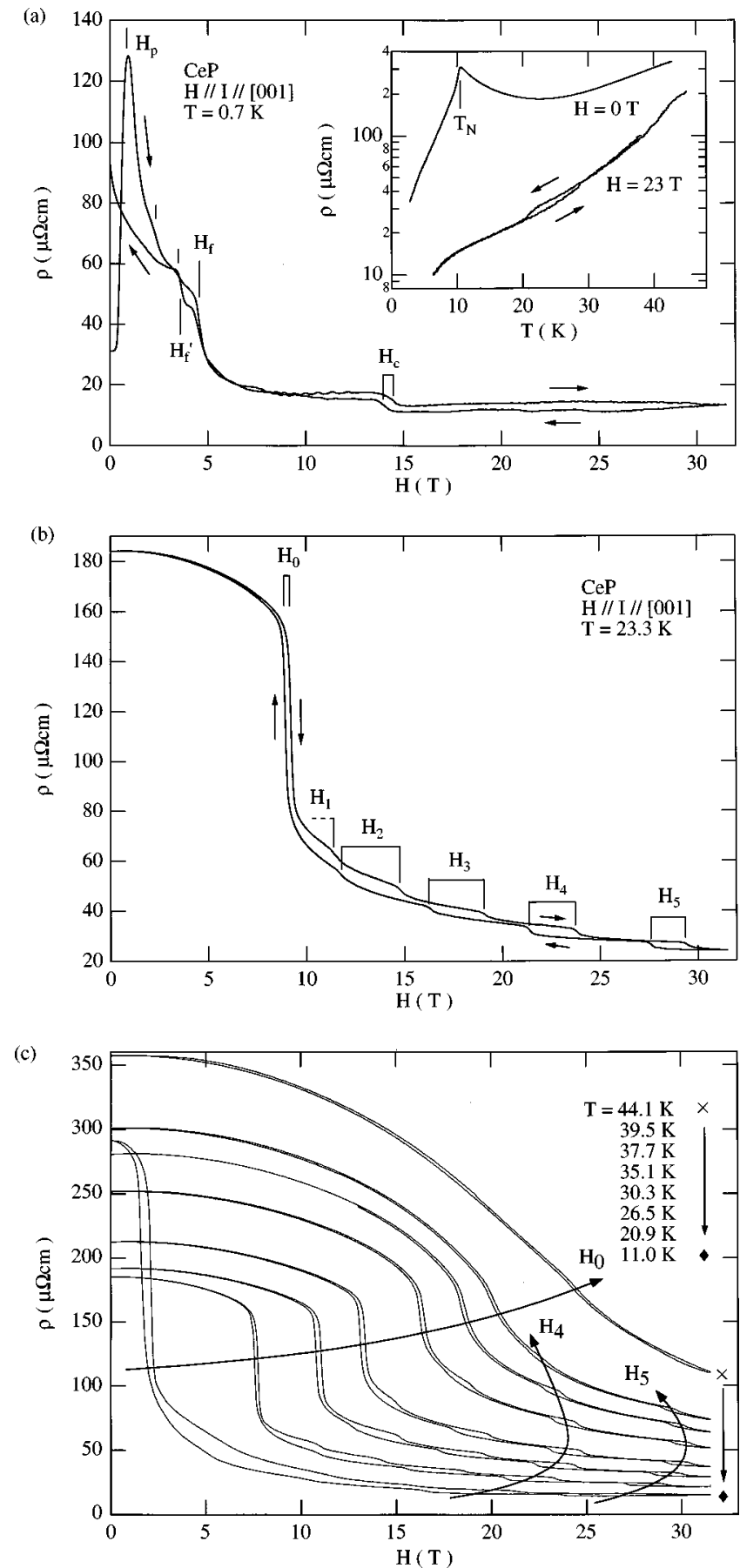

FIG. 2. Resistivity of $\mathrm{CeP}$ as a function of the magnetic field at (a) $T=0.7 \mathrm{~K}$, (b) $T=23.3 \mathrm{~K}$, and (c) several temperatures above $T_{N}=10.5 \mathrm{~K}$. The magnetic field and the electric current were parallel and applied along the [001] direction. The curves $H_{0}, H_{4}$, and $\mathrm{H}_{5}$ shown in the figure (c) are guides to the eye. The inset of the figure (a) shows temperature dependence of the resistivity at constant fields.

transitions, I, II, $D, E, F$, and $j$, reported by Kuroda et al. are also shown by dotted lines. ${ }^{8}$ Our $H_{0}$ transition most likely corresponds to the transition I, though two branches diverging from the phase boundary I, the transition II, and the other one starting at about $34 \mathrm{~K}$, were not observed in the present study. Our $\mathrm{H}_{2}, \mathrm{H}_{3}, \mathrm{H}_{4}$, and $\mathrm{H}_{5}$ transitions seem to correspond to the transitions $j, F, E$, and $D$, respectively.

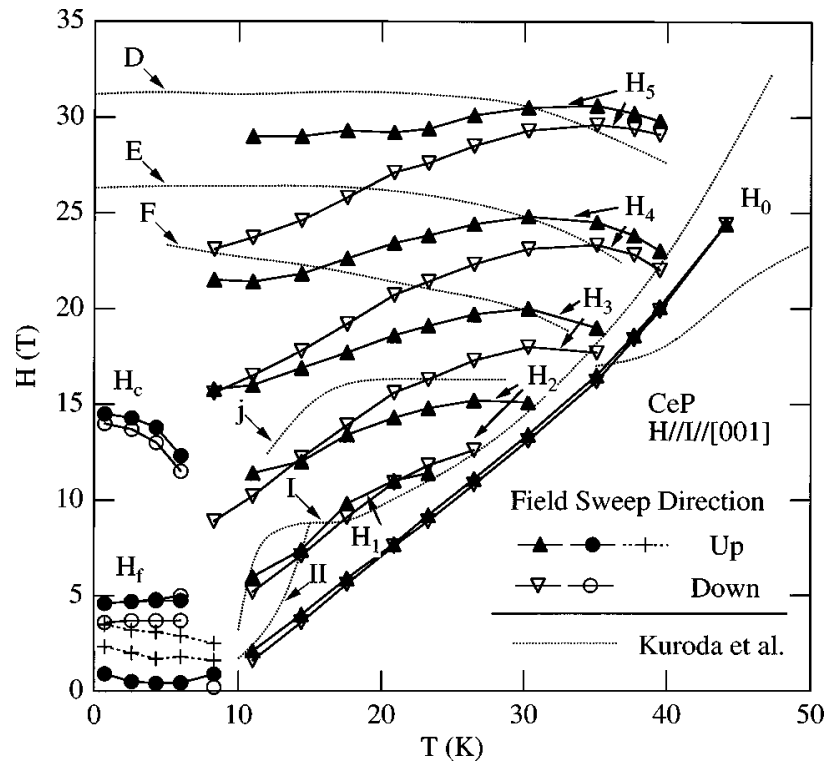

FIG. 3. Transition fields determined from resistivity vs magnetic field traces. The closed symbols and crosses indicate the up-sweep data, while the open symbols show the down-sweep data. The transition fields determined by Kuroda et al. (Ref. 8) are shown by dotted lines for comparison.

Figure 4 shows the inverse transition fields $1 / H_{1}-1 / H_{5}$ at $11.0 \mathrm{~K}$ against an integer index (with arbitrary zero). Since the hysteresis is large, we have plotted not only the up- and down-sweep data but also their medians, which are expected to be good approximation to the thermodynamic transition fields. The transitions $A-F$ reported by Kuroda et al. are also shown for comparison (they observed three transitions $A, B$, and $C$ above the transition $D) .{ }^{8}$ Clearly, our data do not lie on a straight line, and do not support the inverse field relationship.

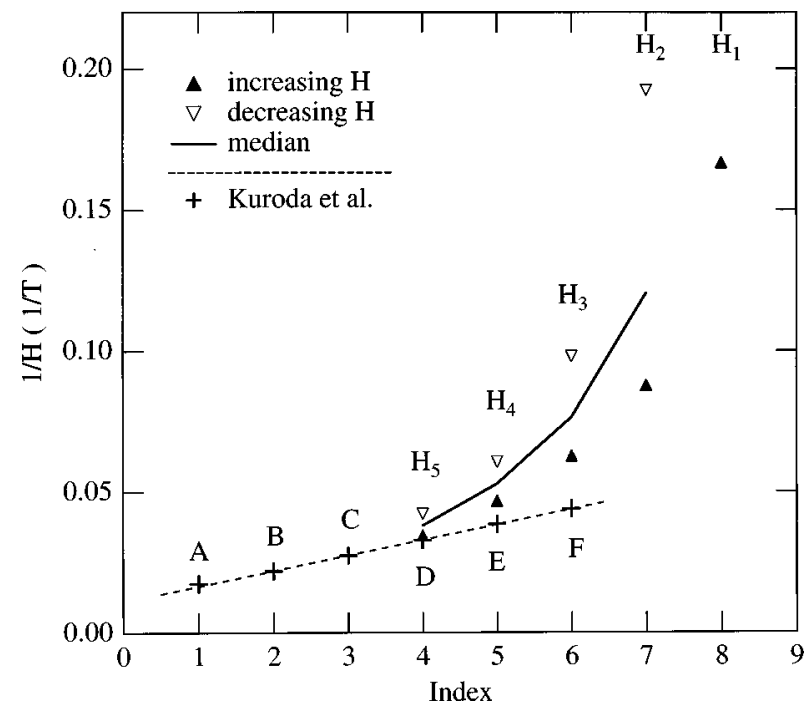

FIG. 4. Inverses transition fields, $1 / H_{0}-1 / H_{5}$, at $11 \mathrm{~K}$ against an integer index (with arbitrary zero). The closed (open) triangles indicate the up-field-sweep (down-field-sweep) data. The solid line shows the medians of the up- and down-sweep transition fields. The inverses transition fields, $1 / A-1 / F$, determined by Kuroda et al. (Ref. 8) are also shown for comparison. 


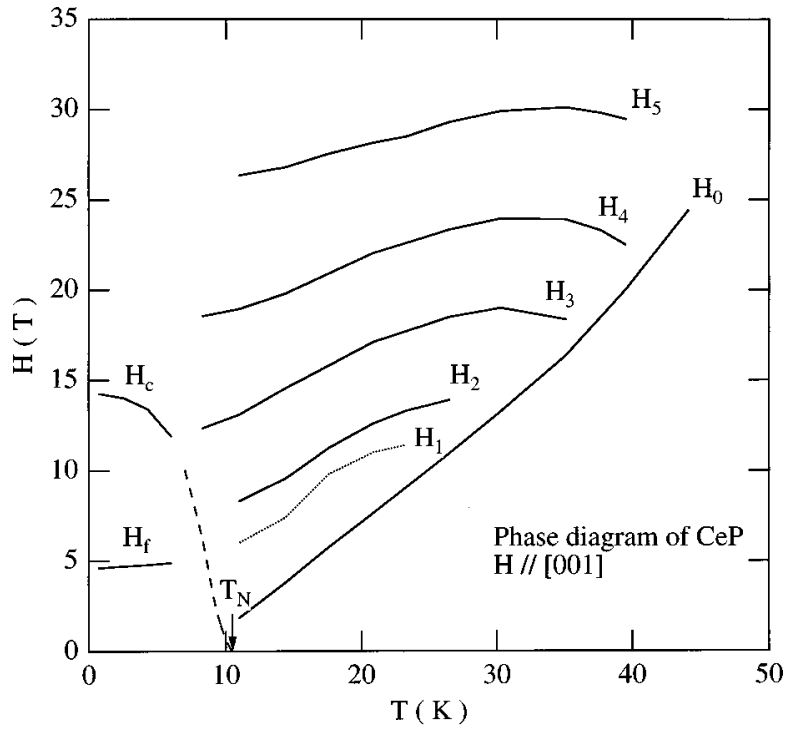

FIG. 5. Magnetic phase diagram of $\mathrm{CeP}$ for the magnetic field parallel to the [001] axis based on the medians of the up- and down-field-sweep transition fields. Since the $H_{1}$ transition is not observed in a down sweep, the up-sweep transitions are shown by the dotted line. The broken line determined from low-field magnetization is reproduced from Ref. 12 .

Figure 5 shows the $H-T$ phase diagram based on the medians of the up- and down-sweep transition fields. Since the $H_{1}$ transition is not observed in the down sweeps, the upsweep data are shown by a dotted line. Some transitions or anomalies are omitted for simplicity. The broken line determined from low-field magnetization data is reproduced from Ref. 12.

\section{DISCUSSION}

The discrepancy between the data of Kuroda et al. (Ref. 8) and ours is appreciable, especially at low temperatures (Fig. 3). We note that Kuroda et al. determined the transition fields based only on up sweeps of the pulsed-magnetic field. Since their transition lines deviate upward from ours at low temperatures, part of the discrepancy is attributed to the difference in sweep rates of the magnetic field. It is possible that the rise of the pulsed magnetic field is too fast compared with the magnetic relaxation of the sample. Another noticeable discrepancy is that we did not see well-developed transitions above $H_{c}$ below $T_{N}$ where Kuroda et al. observed the transitions $D$ and $E$. This point will be discussed later. The most important manifestation of the discrepancy is that the inverses of our transition fields are not equally spaced (Fig. 4). Therefore, our data do not support the idea that the successive metamagnetic transitions are directly connected with the crossing of up- and down-spin Landau levels in a specific conduction-electron energy band.

The magnetoresistance traces observed below $T_{N}$ [Fig. 2(a)] are compatible with previous works on magnetization, ac susceptibility, dHvA effect, and neutron scattering. The magnetization data at $4.2 \mathrm{~K}$ indicate that the transition from the zero-field antiferromagnetic (AF) state to phase I is accompanied by a gradual increase in the magnetization between about 0.1 and $1 \mathrm{~T}$ rather than a sharp jump. ${ }^{12}$ The initial steep increase in the resistivity is probably due to disorder introduced by random nucleation of phase I domains. The peak field $H_{p}$ may thus be assigned to the field where the gradual transformation finishes. Then the domains merge into larger ones and the magnetic ordering becomes more perfect. This could be an origin of the subsequent decrease in the resistivity. The magnetization data also indicate that, after application of the magnetic field, phase I instead of the AF state stays stable in zero-magnetic field. ${ }^{12}$ Thus the lack of the resistivity peak in the down-sweep trace is also in line with the magnetization data. The $H_{f}$ transition is attributed to the spin-flop transition in the $\mathrm{AF} \Gamma_{7}$ layers as was demonstrated by the neutron-scattering experiments. ${ }^{6}$ Then the $H_{c}$ transition can be ascribed to the transition to the fieldinduced ferromagnetic state of the $\Gamma_{7}$ layers. The ac susceptibility data support the interpretation (Ref. 10): it drops at the $H_{c}$ transition as is usually observed in an antiferromagnet when a field-induced ferromagnetic state is stabilized over a spin-flop state with increasing field. The most remarkable feature in Fig. 2(a) is the fact that the up- and down-sweep traces never coincide with each other. This is in accord with unusually history-sensitive dHvA oscillations reported previously. ${ }^{9}$ The dHvA oscillations in an up sweep of the field and those in a subsequent down sweep were found to be very different.

The absence of the transitions $H_{1}-H_{5}$ below $T_{N}$ in our data can be justified by taking account of the extraordinarily strong hysteresis. The strong hysteresis implies substantial energy barriers between different magnetic phases. Since there is a definite difference between the up-sweep and down-sweep traces of the magnetoresistance above $H_{c}$ [Fig. 2(a)], it is apparent that different magnetic phases are nucleated above $H_{c}$ even below $T_{N}$. However, because of the large energy barrier, the local nucleation of a magnetic phase can not develop into a full phase transition over the whole crystal. That is, the transitions are smeared out by the hysteresis. The ac susceptibility data provide indications of such nucleations. Several sharp spikes have been recorded in lowtemperature $(T=0.05 \mathrm{~K})$ ac susceptibility measurements. ${ }^{10}$ The fields where the spikes appear differ each time. They could be due to the local nucleation of magnetic phases. The question why Kuroda et al. observed the successive transitions even below $T_{N}$ remains to be answered. One possible explanation could be sample heating due to the quick rise of the pulsed field.

The phase boundaries $H_{1}-H_{5}$ in the present phase diagram (Fig. 5) quite resembles the phase boundaries between FP phases of $\mathrm{CeSb}$ (Ref. 2). The magnetic structure in the FP phases is characterized by regular stacking of ferromagnetic and paramagnetic layers. With increasing field, stacking sequences with a denser ferromagnetic-layer content appear in order. The phase boundaries between those phases are approximately parallel in the $H-T$ plane. In the case of $\mathrm{CeP}$, a regular lattice of the ferromagnetic $\Gamma_{8}$ layers forms in the sea of the paramagnetic $\Gamma_{7}$ layers above $H_{0}$ (the phase III region). By analogy to the CeSb FP phases, we assume that the transitions $H_{1}-H_{5}$ correspond to discontinuous increases in the $\Gamma_{8}$ to $\Gamma_{7}$ ratio. Then general features of the phase boundaries $H_{0}-H_{5}$ are well explained by a simple thermodynamic model that is essentially the same that applied by RossatMignod et al. to explain the complex phase diagram of 
$\mathrm{CeSb} .{ }^{2}$ We attribute given magnetic moment $M_{7}\left(M_{8}\right)$ and entropy $S_{7}\left(S_{8}\right)$ to a $\Gamma_{7}\left(\Gamma_{8}\right)$ spin. The slopes of the phase boundaries are determined by the formula $d H / d T=-\Delta S / \Delta M$, where $\Delta S=S_{8}-S_{7}$ and $\Delta M=M_{8}-M_{7}$. Letting $S_{7}=k \ln 2$ and $M_{7}=0$ since the $\Gamma_{7}$ spins are paramagnetic, and letting $S_{8}=0$ and $M_{8}=2 \mu_{B}$ since the $\Gamma_{8}$ spins are ferromagnetically ordered, we have $d H / d T=0.59 \mathrm{~T} / \mathrm{K}$, which is close to the observed slope of the $H_{0}$ transition, $0.52 \mathrm{~T} / \mathrm{K}$ in the limit of $H \rightarrow 0$. This simplified model predicts the same slope for all the phase boundaries $H_{0}-H_{5}$, while the slopes of the observed phase boundaries become smaller at higher fields. This is because $\Delta S$ becomes zero before $\Delta M: \Delta M$ stays finite even when the $\Gamma_{7}$ spins are fully aligned by the magnetic field and hence $S_{7}=0$. One important feature that the model fails to explain is the negative slopes of $H_{3}, H_{4}$, and $H_{5}$ above about $30 \mathrm{~K}$. The local maxima of the phase boundary lines indicate that the entropies of the $\Gamma_{8}$ and $\Gamma_{7}$ spins are comparable at this temperature regime. We may ascribe this to excitations within the $\Gamma_{8}$ state that may no longer be negligible. A quantitative argument is, however, not possible until the stabilization mechanism of the $\Gamma_{8}$ state is clarified.

Since the $H-T$ and pressure versus temperature phase diagrams of $\mathrm{CeP}$ are known to be very similar, ${ }^{4}$ it is interesting to note results of high-pressure neutron-scattering experiments. The magnetic structures $9^{\circ}, 8^{\circ}, 7^{\circ}$, and so on appear in order with increasing pressure, ${ }^{13}$ where the structure $n^{\circ}$ has an $n$-layer period composed of a ferromagnetic $\Gamma_{8}$ double layer and $(n-2)$ paramagnetic $\Gamma_{7}$ layers. If we apply the same scenario to the $H-T$ phase diagram, the magnetic structure just above $H_{5}$ will be $5^{\circ}$ (note that the magnetic structure just above $H_{0}$ is $10^{\circ}$ ). According to the pulsed-field magnetization measurements, three or four transitions are expected above $H_{5}$ and they are enough to drive the whole spin system into a complete ferromagnetic state (the magnetic structure $2^{\circ}$ ), though one extra spin structure other than the $n^{\circ}$ series is necessary in the case of the four transitions.

\section{CONCLUSION}

The successive metamagnetic transitions in $\mathrm{CeP}$ were clearly observed in the resistivity versus magnetic field and resistivity versus temperature traces. The phase diagram obtained shows considerable disagreement with one previously determined from magnetization measurements in pulsed magnetic fields. The present transition fields are not equally spaced on the $1 / H$ scale, thereby giving strong evidence against the idea that the metamagnetic transitions are directly connected with the Landau-quantization of a specific conduction-electron energy band. The present phase diagram reveals a close relation to the FP phases of $\mathrm{CeSb}$. We can account for the general features of the phase diagram using the simple thermodynamic model that is essentially the same as previously applied to $\mathrm{CeSb}$. We have speculated that the magnetic structure will change from $10^{\circ}$ to $9^{\circ}, 8^{\circ}$, and so on with increasing magnetic field. It would be very interesting that such changes are confirmed by neutron-scattering experiments in pulsed magnetic fields. The local maxima of the phase boundary lines appearing around $30 \mathrm{~K}$ at high fields are worthy of further investigations in relation to the magnetic polaron model of $\mathrm{CeP}$. It could provide valuable information about how the magnetic polaron evolves with the temperature.
*Present address: National High Magnetic Field Laboratory, Tallahassee, Florida 32310 (on leave from NRIM).

${ }^{1}$ T. Kasuya, O. Sakai, J. Tanaka, H. Kitazawa, and T. Suzuki, J. Magn. Magn. Mater. 63\&64, 9 (1987).

${ }^{2}$ J. Rossat-Mignod, P. Burlet, J. Villain, H. Bartholin, Wang Tcheng-Si, D. Florence, and O. Vogt, Phys. Rev. B 16, 440 (1977); J. Rossat-Mignod, J. M. Effantin, P. Burlet, T. Chattopadhyay, L. P. Regnault, H. Bartholin, C. Vettier, O. Vogt, D. Ravot, and J. C. Achart, J. Magn. Magn. Mater. 52, 111 (1985).

${ }^{3}$ Y. S. Kwon, Y. Haga, O. Nakamura, T. Suzuki, and T. Kasuya, Physica B 171, 324 (1990).

${ }^{4}$ For a review, see, T. Suzuki et al., Physica B 206\&207, 771 (1995)

${ }^{5}$ M. Kohgi, Y. Osakabe, N. Môri, H. Takahashi, Y. Okayama, H. Yoshizawa, Y. Ohara, S. Ikeda, T. Suzuki, and Y. Haga, Physica B 186-188, 393 (1993).

${ }^{6}$ M. Kohgi, T. Osakabe, K. Kakurai, T. Suzuki, Y. Haga, and T. Kasuya, Phys. Rev. B 49, 7068 (1994); M. Kohgi, T. Osakabe, T. Suzuki, Y. Haga, T. Kasuya, and Y. Morii, Physica B 206\&207, 783 (1995)

${ }^{7}$ T. Kasuya, T. Suzuki, and Y. Haga, J. Phys. Soc. Jpn. 62, 2549
(1993); T. Kasuya, Y. Haga, T. Suzuki, T. Osakabe, and M. Kohgi, ibid. 62, 3376 (1993).

${ }^{8}$ T. Kuroda, K. Sugiyama, Y. Haga, T. Suzuki, A. Yamagishi, and M. Date, Physica B 186-188, 396 (1993); T. Inoue, T. Kuroda, K. Sugiyama, Y. Haga, T. Suzuki, and M. Date, J. Phys. Soc. Jpn. 64, 572 (1995).

${ }^{9}$ T. Terashima, S. Uji, H. Aoki, W. Joss, Y. Haga, A. Uesawa, and T. Suzuki, Phys. Rev. B 55, 4197 (1997).

${ }^{10}$ T. Terashima, C. Haworth, M. Takashita, S. Uji, H. Aoki, Y. Haga, A. Uesawa, and T. Suzuki, J. Magn. Magn. Mater. 177181, 421 (1998).

${ }^{11}$ B. L. Brandt and D. W. Liu, Rev. Sci. Instrum. (to be published).

${ }^{12}$ Y. Haga, Ph.D. thesis, Tohoku University (1994); T. Suzuki, in Physical Properties of Actinide and Rare Earth Compounds, JJAP series 8, edited by T. Kasuya, T. Ishii, T. Komatsubara, O. Sakai, N. Mori, and T. Saso (Jpn. J. Appl. Physics, Tokyo, 1993), p. 267.

${ }^{13}$ T. Osakabe, M. Kohgi, K. Iwasa, N. Nakajima, J. M. Mignot, I. N. Goncharenko, Y. Okayama, H. Takahashi, N. Môri, Y. Haga, and T. Suzuki, Physica B 230-232, 645 (1997). 